\title{
Efficient Modal Analysis of Arbitrarily Shaped Waveguides Composed of Linear, Circular, and Elliptical Arcs Using the BI-RME Method
}

\author{
Santiago Cogollos, Stephan Marini, Vicente E. Boria, Senior Member, IEEE, Pablo Soto, Ana Vidal, \\ Hector Esteban, Associate Member, IEEE, Jose V. Morro, and Benito Gimeno, Member, IEEE
}

\begin{abstract}
This paper deals with the accurate and efficient modal analysis of arbitrarily shaped waveguides whose cross section is defined by a combination of straight, circular, and/or elliptical arcs. A novel technique for considering the presence of circular and/or elliptical segments within the frame of the well-known boundary integral-resonant mode expansion (BI-RME) method is proposed. This new extended BI-RME method will allow a more accurate solution of a wider number of hollow conducting waveguides with arbitrary profiles, which are usually present in most modern passive waveguide components. To show the advantages of this new extended technique, the modal chart of canonical (circular and elliptical) waveguides, as well as of irises with great practical interest (i.e., cross-shaped irises with rounded corners) has been first successfully solved. Next, a computer-aided-design software package based on such a novel modal analysis tool has first been validated with the accurate analysis of a referenced complex dual-mode filter, and then applied to the complete design of a novel twist component for $K$-band application based on circular and elliptical waveguides. A prototype of this novel device has been manufactured and measured for verification purposes.
\end{abstract}

Index Terms-Green functions, integral equations, waveguide components, waveguides.

\section{INTRODUCTION}

$\mathbf{O}$ VER THE LAST two decades, an increasing number of passive waveguide devices have been made of waveguides with an arbitrary cross section defined by linear, circular, and/or elliptical arcs. For instance, ridged rectangular [1] and ridged circular [2] waveguides, as well as cross-shaped irises [3] are frequently found in dual-mode empty or dielectric-loaded resonator filters. Multiridged rectangular waveguides have been also employed as tuning elements in reentrant coaxial filters [4], as well as key elements in doubly corrugated chokes [5]. Recently, and due to the mechanization effects of most common manufacturing techniques of waveguide components, the presence of rounded corners in rectangular waveguides has

Manuscript received April 17, 2003. This work was supported by the European Commission under the Research and Training Networks Programme Contract HPRN-CT-2000-00043, and by the Ministerio de Ciencia y Tecnología, Spanish Government, under Research Project TIC2000-0591-C03-01 and Research Project TIC2000-0591-C03-03.

S. Cogollos, S. Marini, V. E. Boria, P. Soto, A. Vidal, H. Esteban, and J. V. Morro are with the Departamento de Comunicaciones, Universidad Politécnica de Valencia, E-46022 Valencia, Spain (e-mail: vboria@dcom.upv.es).

B. Gimeno is with the Departamento de Física Aplicada-Instituto de Ciencia de los Materiales, Universidad de Valencia, E-46100 Burjassot, Valencia, Spain. Digital Object Identifier 10.1109/TMTT.2003.819776 been under investigation in both guided [6] and radiating applications [7]. Another example of great practical interest is the elliptical waveguide, which has found increasing application in many passive microwave components, such as dual-mode [8] and triple-mode [9] filters, circular waveguide polarizers [10], radiators [11], resonators [12], and corrugated horns [13].

Therefore, the electromagnetic-wave propagation in hollow conducting waveguides of arbitrary cross section has become a problem of considerable practical interest, and many different approaches dealing with the calculation of the full modal spectrum of such waveguides have been published in the technical literature. A very early contribution can be found in [14], where a conformal transformation technique is proposed for the study of rectangular waveguides with trapezoidal and semicircular ridges. Over the following years, several techniques were introduced in order to cope with the efficient modal computation of particular arbitrarily shaped waveguides such as nonsymmetric uniform waveguides or triangular- and star-shaped guides. An interesting review of such techniques can be found in [15] and [16].

In the decade of the 1980s, several new techniques for solving the modal problem under consideration were proposed. They can be grouped into two main categories: the first one based upon the solution of integral equations through different methods (see, for instance, [17]-[19]) and the second one consisting of meshing techniques such as the transmission-line modeling method [20] and the finite-element method [21]. Even though the first group of techniques has recently been revisited with the proposition of novel methods, e.g., the generalized spectral-domain method [22] and boundary integral-equation method [23], they lead to the solution of small-size nonalgebraic eigenvalue problems, which, in some cases, do require time-consuming procedures for searching the required cutoff frequencies. On the other hand, the meshing methods lead to either large-size standard eigenvalue matrix problems or multistep iterative strategies, thus demanding high computational efforts and/or large computer memory resources.

To overcome these drawbacks, a new algorithm also based on the solution of an integral equation was originally proposed in [24], i.e., the well known boundary integral-resonant mode expansion (BI-RME) method. The main advantage of this new integral equation method is that it leads to small-size linear matrix eigenvalue problems, which can be accurately solved in rather short CPU times. Recently, the BI-RME method has been revisited in order to also provide the modal coupling coefficients 
of step discontinuities involving arbitrarily shaped waveguides (see [25] and [26]), which has eased its practical integration into modern computer-aided design (CAD) tools [27]. Nevertheless, despite these recent efforts devoted to improving the BI-RME method, all practical implementations of such a technique use only straight segments for describing the arbitrarily shaped contours, even though they are composed of circular and/or elliptical arcs. This simple approach, which usually requires a higher number of straight segments to define the arbitrary profiles, does not provide enough accurate results for some restrictive practical applications.

Within the context of this high demanding scenario, this paper is essentially aimed at describing a rigorous method that allows the accurate consideration of linear, circular, and/or elliptical arcs by the BI-RME formulation, as well as the appropriate connection of such types of segments. To fully validate the new theory proposed in this paper, two simple canonical examples have first been considered. One of them is a circular waveguide and the other is an elliptical guide, whose modal charts are either analytically or numerically well known. After this successful preliminary verification, the new theory proposed has been applied to the complete modal analysis of a commonly used iris, i.e., the cross-shaped iris, but considering rounded corners due to mechanization effects. Next, the new modal analysis tool developed has been integrated into a CAD software package of complex passive waveguide devices. Such CAD package has been first verified with the accurate analysis of a dual-mode filter involving circular and elliptical waveguides. Finally, making use of the validated CAD tool, a novel topology for a $K$-band $90^{\circ}$-twist component based on circular and elliptical waveguides has been proposed. The simulated results of this new component have been successfully compared with measurements of a manufactured prototype. The computational efficiency of the novel modal analysis tool, as well as of the CAD software package based on such a tool, has been revealed as being very good.

\section{THEORY}

The structure under investigation is the arbitrarily shaped waveguide shown in Fig. 1, whose cross section $S$ can be defined by a combination of linear, circular, and/or elliptical arcs. As can be seen in this figure, the arbitrary cross section is enclosed within a standard rectangular waveguide $\Omega$, and its arbitrary contour $\sigma$ is defined by the tangent vector $\hat{\mathbf{t}}$ and the suitable abscisa $l$ taken on the contour line.

In order to obtain the modal chart of such arbitrary waveguides, the already cited BI-RME method, first described in [24], is proposed. The practical implementations of this classical technique, as well as of further revisited versions of the method (see, for instance, [27]), always have the arbitrary profile $\sigma$ segmented into smaller straight arcs. In this section, we will only present the new theoretical aspects related to the BI-RME method implementation that are needed to also consider circular and/or elliptical arcs when segmenting the arbitrarily shaped contours. A detailed explanation of the generic BI-RME method formulation, and also of its classical implementation, can be found in [24] and [27].

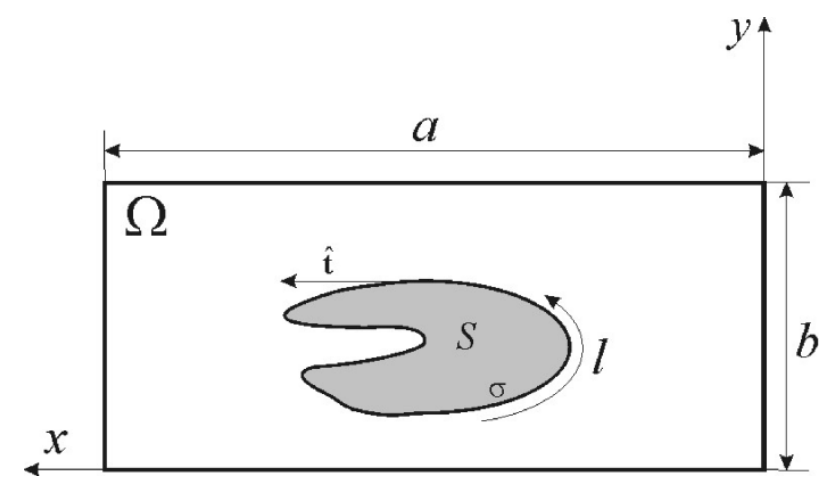

Fig. 1. Waveguide with an arbitrary cross section $S$ enclosed within a standard rectangular waveguide $\Omega$.

A practical procedure for efficiently solving the connection of the two new kinds of arcs introduced (i.e., the circular and elliptical ones) with the standard straight segments used up to now in the classical BI-RME implementation will also be outlined.

Once the modal chart of an arbitrarily shaped waveguide including straight, circular, and/or elliptical arcs is solved, the efficient and accurate procedures described in [25] and [26] can also be followed in order to easily compute the modal coupling coefficients of such modes with those of the standard rectangular waveguide enclosing the arbitrary profile (see Fig. 1).

\section{A. Extension of the TM Case}

When computing the TM modes of an arbitrarily shaped waveguide using the BI-RME method originally described in [24], the most crucial task is related to the accurate evaluation of the following matrix elements:

$$
L_{i j}^{\prime}=\int_{\sigma} \int_{\sigma} u_{i}(l) g\left(\mathbf{s}, \mathbf{s}^{\prime}\right) u_{j}\left(l^{\prime}\right) d l d l^{\prime}
$$

where the functions $u_{i}$ and $u_{j}$ are the basis and testing functions related to the implementation of the well-known method of moments (MoM). Typically [24], such functions are piece-wise parabolic splines defined in two or three segments of the arbitrary contour, which, in our case, can be straight, circular, and/or elliptical arcs. In each of these segments, these functions have the following simple expression:

$$
u(l)=a l^{2}+b l+c
$$

where the coefficients $a, b$, and $c$ are explicitly reported in [27] for the cases of straight and circular segments. If an elliptical arc is involved, these coefficients must be computed following the procedure described at the end of this section.

In (1), $\mathbf{s}$ and $\mathbf{s}^{\prime}$ are, respectively, the source and field vectors addressing points of the arbitrary contour $\sigma$, which can be defined as $\sigma=\mathbf{s}(l)$, and $g$ is the scalar two-dimensional Green function for the Poisson equation [24].

When $i \neq j$, the double integral defined in (1) can be performed numerically in a very easy way, for instance, using a Gauss quadrature rule. However, such an approach cannot be followed with the diagonal elements of the $\mathbf{L}^{\prime}$ matrix (i.e., when $i=j$ ) since $g$ is singular when $\mathbf{s}=\mathbf{s}^{\prime}$. 
A rapidly convergent expression for such a Green function can be found in [28], which has the following aspect:

$$
g=\frac{1}{4 \pi} \sum_{m=-\infty}^{\infty} \ln \frac{T_{m}^{10} T_{m}^{01}}{T_{m}^{00} T_{m}^{11}}
$$

where

$$
\begin{aligned}
T_{m}^{p q} & =1-2 e^{\tau_{m p}} \cos \frac{\pi}{b}\left(y-(-1)^{q} y^{\prime}\right)+e^{2 \tau_{m p}} \\
\tau_{m p} & =-\left|x-(-1)^{p} x^{\prime}+2 a m\right| \frac{\pi}{b} .
\end{aligned}
$$

It should be noticed that the singularity of the $g$ function is due to the $\ln T_{0}^{00}$ term, which tends to infinity when the field point $\mathbf{s}(x, y)$ approaches the source point $\mathbf{s}^{\prime}\left(x^{\prime}, y^{\prime}\right)$. Under such circumstances, the $\ln T_{0}^{00}$ behaves like the singular function $\ln R$, where $R$ denotes the Cartesian distance between the aforementioned field and source points.

In order to treat the singular behavior of the $g$ function, the singular term of such a function already detected must first be isolated. Next, to simplify the expression of this problematic term, a well-known technique for solving generic integral equations with singularities will be followed [29]. This classical technique, which essentially consists of adding and subtracting a canonical function with the same kind of singularity behavior to the singular term, has already been successfully used together with the MoM approach [30].

Therefore, making use of such a classical technique, our original scalar two-dimensional Green function $(g)$ can be split as follows:

$$
g=g_{r}+g_{r s}+g_{s}
$$

with

$$
\begin{aligned}
g_{r} & =\frac{1}{4 \pi} \sum_{\substack{m=-\infty \\
(m \neq 0)}}^{\infty} \ln \frac{T_{m}^{10} T_{m}^{01}}{T_{m}^{00} T_{m}^{11}}+\frac{1}{4 \pi} \ln \frac{T_{0}^{10} T_{0}^{01}}{T_{0}^{11}} \\
g_{r s} & =-\frac{1}{4 \pi} \ln \frac{T_{0}^{00}}{R^{2}} \\
g_{s} & =-\frac{1}{4 \pi} \ln R^{2} .
\end{aligned}
$$

In (5) and (6), the subscript $r$ denotes the completely regular contribution of the scalar Green function, the compound subscript $r s$ indicates that the singular term $\ln T_{0}^{00}$ has been regularized using the well-known technique just previously outlined, and finally, the subscript $s$ refers to the isolated singular term of the $g$ function, which is expressed as a canonical function whose singularity can be analytically treated.

With regard to the regularized term $g_{r s}$ of the scalar Green function, its regular value when the field and source points are very close to each other can be easily obtained by expanding the $T_{0}^{00}$ function as a Taylor series and then taking the corresponding limit. Proceeding in such a way, it is finally obtained that

$$
\lim _{\substack{x \rightarrow x^{\prime} \\ y \rightarrow y^{\prime}}} \frac{T_{0}^{00}}{\left(x-x^{\prime}\right)^{2}+\left(y-y^{\prime}\right)^{2}}=\left(\frac{\pi}{b}\right)^{2} .
$$

The only contribution to the $L_{i i}^{\prime}$ matrix elements that remains to be treated is then the one related to the singular term $\left(g_{s}\right)$ of the Green function. With the aim of making such double-integral

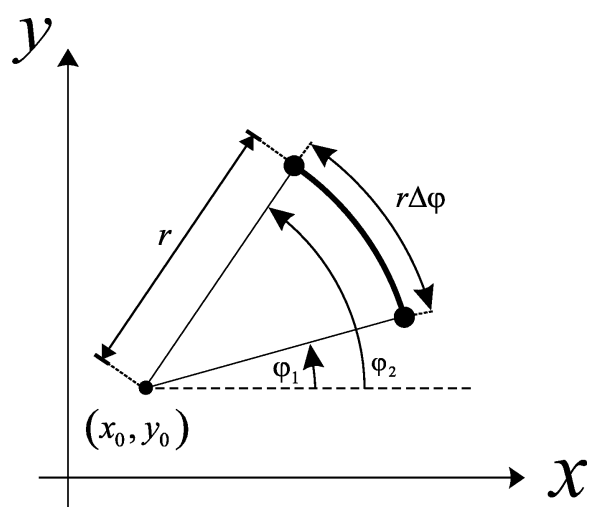

Fig. 2. Arbitrarily oriented circular arc with radius $r$ and length $r \Delta \varphi$.

contribution independent of the kind of arcs under consideration (straight, circular, and elliptical ones), such integration will be always performed in the same interval $[-0.5,0.5]$ of a dummy parameter $t$ to be suitably defined in each case. In fact, the only integral to be solved analytically will be the inner one, whereas the remaining outer one will finally be computed numerically following a simple Gauss quadrature rule.

Next, we offer further mathematical details regarding the practical application of the above-described technique to the particular cases of circular and elliptical arcs.

1) Circular Arcs: A circular arc (see Fig. 2) can be easily described in terms of a $t$-parameter running in the interval $[-0.5,0.5]$ as follows:

$$
\begin{aligned}
& x=x_{0}+r \cos \varphi(t) \\
& y=y_{0}+r \sin \varphi(t)
\end{aligned}
$$

where

$$
\begin{aligned}
\varphi(t) & =\varphi_{1}+\Delta \varphi(t+0.5) \\
\Delta \varphi & =\varphi_{2}-\varphi_{1}
\end{aligned}
$$

and $r$ is the constant radius of the circular arc.

Inserting these previous equations within the definition of the singular term $\left(g_{s}\right)$ of the Green function in (6), such a term can now be easily divided into the following two components:

$$
g_{s}=g_{s r}+g_{s s}
$$

where

$$
\begin{aligned}
& g_{s r}=-\frac{1}{4 \pi} \ln \frac{R^{2}}{\Delta \varphi^{2}\left(t-t^{\prime}\right)^{2}} \\
& g_{s s}=-\frac{1}{4 \pi} \ln \left[\Delta \varphi^{2}\left(t-t^{\prime}\right)^{2}\right] .
\end{aligned}
$$

Now, $g_{s r}$ can also be treated as a regular function because

$$
\lim _{\substack{x \rightarrow x^{\prime} \\ y \rightarrow y^{\prime}}} \ln \frac{R^{2}}{\Delta \varphi^{2}\left(t-t^{\prime}\right)^{2}}=\ln r^{2}
$$

and, therefore, the contribution of such a term to the $L_{i i}^{\prime}$ matrix elements will be also computed numerically.

With regard to the inner singular integral of the $L_{i i}^{\prime}$ matrix elements related to the term $g_{s s}$ just outlined in (11), an analytical solution is explicitly detailed in (38) of the Appendix .

2) Elliptical Arcs: In this case, an elliptical arc, shown in Fig. 3, must be described in terms of a $t$-parameter running in the 


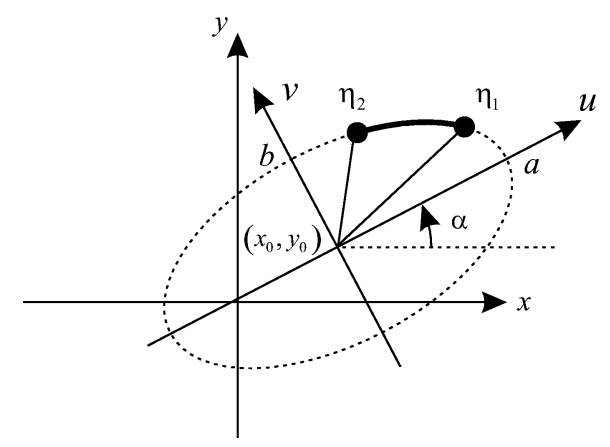

Fig. 3. Arbitrarily oriented elliptic arc defined by the angles $\eta_{1}$ and $\eta_{2}$ of an ellipse with major and minor semiaxes $a$ and $b$.

interval $[-0.5,0.5]$. First, the elliptic arc is described in terms of a more suitable local coordinate system (see Fig. 3) as follows:

$$
\begin{aligned}
& u=a \cos \eta(t) \\
& v=b \sin \eta(t)
\end{aligned}
$$

where

$$
\begin{aligned}
\eta(t) & =\eta_{1}+\Delta \eta(t+0.5) \\
\Delta \eta & =\eta_{2}-\eta_{1} .
\end{aligned}
$$

Next, the local coordinate system chosen before must be related to the global Cartesian system defined by the $x$ and $y$ coordinates. To do so, the following relationship between both coordinate systems must be considered:

$$
\left(\begin{array}{l}
x \\
y
\end{array}\right)=\left(\begin{array}{l}
x_{0} \\
y_{0}
\end{array}\right)+\left(\begin{array}{cc}
\cos \alpha & -\sin \alpha \\
\sin \alpha & \cos \alpha
\end{array}\right) \cdot\left(\begin{array}{l}
u \\
v
\end{array}\right) .
$$

Introducing all these previous relations within the expression of the singular term $g_{s}$ outlined in (6), such a term can now be easily divided again into the following two new components:

$$
g_{s}=g_{s r}+g_{s s}
$$

where now

$$
\begin{aligned}
& g_{s r}=-\frac{1}{4 \pi} \ln \frac{R^{2}}{\Delta \eta^{2}\left(t-t^{\prime}\right)^{2}} \\
& g_{s s}=-\frac{1}{4 \pi} \ln \left[\Delta \eta^{2}\left(t-t^{\prime}\right)^{2}\right] .
\end{aligned}
$$

The $g_{s r}$ term can be considered again as a regular function since, in this case,

$$
\lim _{\substack{x \rightarrow x^{\prime} \\ y \rightarrow y^{\prime}}} \ln \frac{R^{2}}{\Delta \eta^{2}\left(t-t^{\prime}\right)^{2}}=\ln \left[a^{2} \sin ^{2} \eta(t)+b^{2} \cos ^{2} \eta(t)\right]
$$

and, therefore, the contribution of such a term to the $L_{i i}^{\prime}$ matrix elements can also be performed numerically.

With regard to the inner singular integral of the $L_{i i}^{\prime}$ matrix elements related to the term $g_{s s}$ just presented above in (17), a further refined treatment is needed in order to reach a kind of singular integral like the one solved in (38).

Such further refined treatment is needed due to the fact that the expressions for the length differentials present in $L_{i i}^{\prime}$ matrix elements, i.e., $d l$ and $d l^{\prime}$ in (1), do have more complicated expressions in terms of the dummy parameters $t$ and $t^{\prime}$ than those obtained for the circular case. For instance, using the expressions collected in (13) and (14), the length differential $d l$ for an elliptical arc is defined as follows:

$$
d l=a \Delta \eta \sqrt{1-e^{2} \cos ^{2} \eta(t)} d t
$$

where $e$ is the eccentricity of the ellipse where the elliptical arc is integrated.

If this definition of the length differential is considered within the generic expression of the $L_{i i}^{\prime}$ matrix elements, the following double integral is finally obtained:

$$
L_{i i}^{\prime}=a^{2} \Delta \eta^{2} \int_{-0.5}^{0.5} \int_{-0.5}^{0.5} u_{i}(t) \gamma(t) g\left(\mathbf{s}, \mathbf{s}^{\prime}\right) u_{j}\left(t^{\prime}\right) \gamma\left(t^{\prime}\right) d t d t^{\prime}
$$

where $\gamma(t)=\sqrt{1-e^{2} \cos ^{2} \eta(t)}$.

Now, we can make use of the decomposition of the scalar Green function $g$ previously proposed in this section. Proceeding in this way, the computation of (20) can be split into the following two terms:

$$
L_{i i}^{\prime}=L_{i i r}^{\prime}+L_{i i s}^{\prime}
$$

where

$$
\begin{aligned}
& L_{i i r}^{\prime}=a^{2} \Delta \eta^{2} \int_{-0.5}^{0.5} \int_{-0.5}^{0.5} u_{i}(t) \gamma(t)\left(g_{r}+g_{r s}+g_{s r}\right) u_{j}\left(t^{\prime}\right) \gamma\left(t^{\prime}\right) d t d t^{\prime} \\
& L_{i i s}^{\prime}=a^{2} \Delta \eta^{2} \int_{-0.5}^{0.5} \int_{-0.5}^{0.5} u_{i}(t) \gamma(t) g_{s s}\left(t, t^{\prime}\right) u_{j}\left(t^{\prime}\right) \gamma\left(t^{\prime}\right) d t d t^{\prime}
\end{aligned}
$$

In expressions (21) and (22), the subscript $r$ means a regular contribution to the $L_{i i}^{\prime}$ matrix elements, which can, therefore, be computed numerically. On the other hand, the subscript $s$ makes reference to the fact that the related integral is singular, thus needing a special treatment.

For solving the singular integral $L_{i i s}^{\prime}$, a second subdivision level is required, thus giving place to the following decomposition:

$$
L_{i i s}^{\prime}=L_{i i s r}^{\prime}+L_{i i s s}^{\prime}
$$

where now

$$
\begin{aligned}
L_{i i s r}^{\prime}= & a^{2} \Delta \eta^{2} \int_{-0.5}^{0.5} \int_{-0.5}^{0.5} u_{i}(t) \gamma(t)\left(1-\frac{\gamma(t)}{\gamma\left(t^{\prime}\right)}\right) \\
& \times g_{s s}\left(t, t^{\prime}\right) u_{j}\left(t^{\prime}\right) \gamma\left(t^{\prime}\right) d t d t^{\prime} \\
L_{i i s s}^{\prime}= & a^{2} \Delta \eta^{2} \int_{-0.5}^{0.5} \int_{-0.5}^{0.5} u_{i}(t) \gamma^{2}(t) g_{s s}\left(t, t^{\prime}\right) u_{j}\left(t^{\prime}\right) d t d t^{\prime} \\
= & a^{2} \Delta \eta^{2} \int_{-0.5}^{0.5} u_{i}(t) \gamma^{2}(t) \int_{-0.5}^{0.5} g_{s s}\left(t, t^{\prime}\right) u_{j}\left(t^{\prime}\right) d t d t^{\prime} .
\end{aligned}
$$

The second subscript of this new subdivision again gives a clear explanation of the new terms generated. The first new 
term $L_{i i s r}^{\prime}$ has now turned into a regular one since $\lim _{t \rightarrow t^{\prime}}(1-$ $\left(\gamma(t) / \gamma\left(t^{\prime}\right)\right)=0, g_{s s}$ has a singularity of a logarithmic kind, and as is well known, $\lim _{x \rightarrow 0} x^{\alpha} \ln x=0$ for any value of $\alpha$ greater than zero. On the contrary, the second new term $L_{i i s s}^{\prime}$ is still a singular contribution, but, in this case, the inner integral of such a term is identical to the one derived for the circular case, whose analytical solution can be found again in (38).

It is interesting to notice that the new definitions for the length differentials $d l$ and $d l^{\prime}$ must also be introduced into (1) when computing the $L_{i j}^{\prime}$ matrix elements. However, in such case, no singularity is arisen and, therefore, the double integration required to compute such matrix elements can be easily performed in a numerical way.

\section{B. Extension of the TE Case}

For the TE case, the critical issues related to the application of the original BI-RME formulation (see [24]) are related to the accurate computation of the following matrix elements:

$$
\begin{aligned}
C_{i j} & =\int_{\sigma} \int_{\sigma} \frac{\partial u_{i}(l)}{\partial l} g\left(\mathbf{s}, \mathbf{s}^{\prime}\right) \frac{\partial u_{j}\left(l^{\prime}\right)}{\partial l^{\prime}} d l d l^{\prime} \\
L_{i j} & =\int_{\sigma} \int_{\sigma} u_{i}(l) \hat{\mathbf{t}}(l) \cdot \overline{\mathbf{G}}_{s t}\left(\mathbf{s}, \mathbf{s}^{\prime}\right) \cdot \hat{\mathbf{t}}\left(l^{\prime}\right) u_{j}\left(l^{\prime}\right) d l d l^{\prime} .
\end{aligned}
$$

If the first expression of the last two is compared with the definition of the $L_{i i}^{\prime}$ matrix elements presented in (1), it can be easily noticed that both are very similar, and the only difference is related to the presence of the piecewise parabolic functions or their first derivatives. Therefore, the singularity problems related to the evaluation of (25) with circular and elliptical segments can be solved in the same way proposed earlier for the TM case. Note that, for the TE case, the coefficient $a$ in (38) should be set equal to zero.

With regard to the computation of the diagonal elements of the $\mathbf{L}$ matrix, a new procedure for dealing with the new singularities appearing must be developed since, in this case, such singularities are due to the solenoidal dyadic Green function $\overline{\mathbf{G}}_{s t}$. This dyadic function is composed of four components, i.e., $\bar{G}_{s t x x}, \bar{G}_{s t x y}, \bar{G}_{s t y x}$, and $\bar{G}_{s t y y}$, whose compact expressions are explicitly detailed in [24].

The singularities introduced by the $\bar{G}_{s t x x}$ and $\bar{G}_{\text {styy }}$ components are of the same kind (logarithmic one) considered for the TM case. Therefore, the same procedure for the accurate management of such singularities described earlier can now be also followed. Nevertheless, it must be taken into account that the presence of the unitary vectors $\hat{\mathbf{t}}$ in (26) introduce additional sine and cosine terms in the inner singular integrals to be solved analytically. The explicit analytical solutions for this new inner singular integrals are presented in (39) and (40).

In the TE case, an additional problem appears when computing the diagonal elements of the $\mathbf{L}$ matrix, which is related to the fact that some terms of the four components of the solenoidal dyadic Green function do have an unknown value when the field and source points approach each other. To determine these unknown values, a Taylor-series expansion of each one of these terms must be performed, thus giving place to the following two types of functions:

$$
\begin{aligned}
& \Phi=\frac{\left(x-x^{\prime}\right)^{2}}{\left(x-x^{\prime}\right)^{2}+\left(y-y^{\prime}\right)^{2}} \\
& \Psi=\frac{\left(x-x^{\prime}\right)\left(y-y^{\prime}\right)}{\left(x-x^{\prime}\right)^{2}+\left(y-y^{\prime}\right)^{2}} .
\end{aligned}
$$

The $\Phi$ function appears when the terms of the $\bar{G}_{s t x x}$ and $\bar{G}_{\text {styy }}$ components are expanded into the Taylor series, whereas the $\Psi$ function comes from the Taylor-series expansion of the selected terms of the $\bar{G}_{s t x y}$ and $\bar{G}_{s t y x}$ components. It is interesting to remark that two such functions are not singular, but discontinuous, which means that their limit values when the field and source points are close enough depend on the kind of segment they belong to (in our case, a straight, circular, or elliptical arc).

If the arc is a straight one, the limits of (27) and (28) are easily calculated, thus giving place to the following values for the $\Phi$ and $\Psi$ functions:

$$
\begin{aligned}
& \lim _{\substack{x \rightarrow x^{\prime} \\
y \rightarrow y^{\prime}}} \Phi=\cos ^{2} \theta \\
& \lim _{\substack{x \rightarrow x^{\prime} \\
y \rightarrow y^{\prime}}} \Psi=\frac{1}{2} \sin (2 \theta)
\end{aligned}
$$

where $\theta$ is the slope of the straight arc.

For a circular arc, the limit values of the $\Phi$ and $\Psi$ functions are the following:

$$
\begin{aligned}
& \lim _{\substack{x \rightarrow x^{\prime} \\
y \rightarrow y^{\prime}}} \Phi=\sin ^{2}[\varphi(t)] \\
& \lim _{\substack{x \rightarrow x^{\prime} \\
y \rightarrow y^{\prime}}} \Psi=-\frac{1}{2} \sin [2 \varphi(t)] .
\end{aligned}
$$

For an elliptical arc, the $\Phi$ and $\Psi$ functions do have the following limit values:

$$
\begin{aligned}
& \lim _{\substack{x \rightarrow x^{\prime} \\
y \rightarrow y^{\prime}}} \Phi=\frac{[a \cos \alpha \sin \eta(t)+b \sin \alpha \cos \eta(t)]^{2}}{a^{2} \sin ^{2} \eta(t)+b^{2} \cos ^{2} \eta(t)} \\
& \lim _{\substack{x \rightarrow x^{\prime} \\
y \rightarrow y^{\prime}}} \Psi=\frac{1}{2} \frac{\chi(t) \sin 2 \alpha-a b \sin 2 \eta \cos 2 \alpha}{a^{2} \sin ^{2} \eta(t)+b^{2} \cos ^{2} \eta(t)}
\end{aligned}
$$

where $\chi(t)$ is defined as follows:

$$
\chi(t)=a^{2} \sin ^{2} \eta(t)-b^{2} \cos ^{2} \eta(t) .
$$

\section{Solving the Connection of Straight, Circular, and Elliptical Arcs}

A further step to be studied is the connection of the different kinds of segments considered in this paper (i.e. rectangular, circular, and elliptical) for defining the contour $\sigma$ of the arbitrarily shaped waveguides.

The connection of two straight segments with different orientations, as well as the connection of one straight segment with a circular one or of two circular segments, can be easily implemented since the relationship between the length and arc values is straightforward for the circular case. The problem arises when an elliptical arc is to be connected with the two other kinds of 
arcs. Such difficulty is due to the fact that the length of an elliptical arc is not analytically known and, of course, its value is not directly related to the elliptical arc $\Delta \eta$.

As was already explained earlier in this section, the unknown current of the modal problem to be solved by the BI-RME method is reconstructed using piecewise parabolic functions. In [24], it is proposed that the support of such piecewise functions is defined by two or three segments of the original arbitrary contour, and that the area of such functions over its entire domain (the two or three segments considered) must be equal to one in order to guarantee more stable numerical results.

Let us suppose, without any loss of generality, that one segment of a piecewise parabolic function is an elliptical one, whose length $(l)$ is usually fixed by the segmentation procedure of the arbitrarily shaped contour. A typical value for such fixed length is chosen to be equal to $\lambda_{c} / 4$, where $\lambda_{c}$ is the cutoff wavelength of the highest order mode of interest belonging to the arbitrary waveguide [27]. For an elliptical segment, it is not so simple to define the elliptical arc with only such information about the arc length.

To avoid this situation, the following approach has been adopted. If the elliptical arc belongs to an ellipse of major semiaxis $a$, we propose to choose a value for the elliptical arc length $\Delta \eta$ equal to the quotient of the proposed fixed length $l$ and $a$ (i.e., $\Delta \eta=l / a$ ). It must be noticed that this choice of the $\Delta \eta$ value will not provide a real length for the elliptical arc equal to the wanted value $l$. Nevertheless, once the elliptical segment is defined, the coefficient values of the two or three parabolic functions defining the whole piecewise function are easily determined following a standard normalization procedure (for instance, the one described in [27]). The real area of the whole piecewise function must then be determined as follows:

$$
I=\int_{l 1 \rightarrow l 3} u_{n}(l) d l=\int_{l_{1}} u_{n}^{(1)}(l) d l+\int_{l_{2}} u_{n}^{(2)}(l) d l+\int_{l_{3}} u_{n}^{(3)}(l) d l
$$

where $u_{n}$ means the total piecewise basis function to be built, and $u_{n}^{(i)}$ are the parabolic functions defined on each segment (in this particular case, we have considered three segments to define the support of the complete basis function). In our particular example, the third integral in (36) corresponds to the elliptical arc, and will have the following aspect:

$$
\int_{l_{3}} u_{n}^{(3)}(l) d l=a \Delta \eta \int_{-0.5}^{0.5}\left(a_{n} t^{2}+b_{n} t+c_{n}\right) \sqrt{1-e^{2} \cos ^{2} \eta(t)} d t .
$$

Once (36) is solved, we will see how the value obtained for $I$ is not equal to one due to the fact that the length of the real elliptical arc built is not $l$, as has already been explained. The solution is quite simple: the final coefficient values of all the parabolic functions used to define the total piecewise basis function are obtained by simply dividing the ones previously determined by the $I$ value just computed.

Finally, it is interesting to remark that proceeding in this way with the construction of the elliptical arcs, their lengths will be different depending on the position of such segments within the ellipse. In fact, if the elliptical arc is placed where the tangent unitary vector to the ellipse has a higher variation, its length
TABLE I

RElative ERror IN THE CUTOFF FreQuencies OF A CiRCUlar WAVEGUIDE (OF DIAMETER $9.525 \mathrm{~mm}$ ) COMPUTED USING THE BI-RME METHOD WITH ONLY STRAight SEGMENTS AND With ONLY CIRCUlaR ARCS

\begin{tabular}{c||c|c}
\hline Mode & $\begin{array}{c}\text { Error BI-RME } \\
\text { Type }\end{array}$ & $\begin{array}{c}\text { Error BI-RME } \\
\text { (straight segments) }\end{array}$ \\
\hline (circular arcs) \\
$\mathrm{TM}_{01}$ & $0.160 \%$ & $0.002 \%$ \\
$\mathrm{TM}_{11}$ & $0.206 \%$ & $0.009 \%$ \\
$\mathrm{TM}_{21}$ & $0.207 \%$ & $0.004 \%$ \\
$\mathrm{TM}_{02}$ & $0.320 \%$ & $0.011 \%$ \\
$\mathrm{TM}_{31}$ & $0.439 \%$ & $0.013 \%$ \\
$\mathrm{TM}_{12}$ & $0.585 \%$ & $0.015 \%$ \\
$\mathrm{TM}_{41}$ & $0.284 \%$ & $0.021 \%$ \\
$\mathrm{TM}_{22}$ & $0.526 \%$ & $0.012 \%$ \\
$\mathrm{TE}_{11}$ & --- & $0.035 \%$ \\
$\mathrm{TE}_{21}$ & --- & $0.054 \%$ \\
\hline
\end{tabular}

will be smaller, thus giving way to a finer segmentation that will provide more accurate results.

\section{VALIDATION RESULTS}

In this section, the new above-described theory is completely verified with several examples of great practical interest. The results presented have been grouped into two main blocks: the first one dealing with the modal analysis of arbitrarily shaped waveguides and the second one related to the analysis and design of modern passive devices involving such kind of waveguides. In all the example cases considered, the simulated results have been successfully compared with either numerical and experimental data available in the technical literature or with own measurements of manufactured prototypes.

In order to show the efficiency of the novel modal analysis tool developed, as well as of the CAD software packages based on this tool, CPU times have been included in most of the examples considered. Such computational efforts have been always determined on a Pentium IV platform at $2.4 \mathrm{GHz}$ with 1-GB double date rate random access memory (DDRAM).

\section{A. Modal Analysis of Arbitrarily Shaped Waveguides}

To fully validate the new theory developed for circular arcs, as well as its supposed improved accuracy, we have first performed the modal analysis of a canonical waveguide, i.e., a circular waveguide of diameter $9.525 \mathrm{~mm}$, whose cutoff frequencies are analytically known. To make use of the new theory, such a circular waveguide has been defined as a tubular sheet (see $\sigma$ in Fig. 1) perturbing a standard square waveguide ( $\Omega$ in Fig. 1) of size $9.525 \mathrm{~mm}$. In Table I, a comparison between the relative errors of the first TM and TE cutoff frequencies of the circular waveguide modes, computed using the classical (using only straight segments) and the new extended (using, in 
TABLE II

CUTOFF WAVELENGTHS OF AN ELLIPTICAL WAVEGUIDE $(a=10.0 \mathrm{~mm}$ AND $e=0.5)$ USING THE BI-RME METHOD With ELLIPTICAL ARCS. THE REFERENCE VALUES ARE COLLECTED From [31]

\begin{tabular}{c|c||c|c|c}
\hline Order & $\begin{array}{c}\text { Mode Type } \\
\text { (TE/TM) }\end{array}$ & $\begin{array}{c}\lambda_{c}(\mathrm{~cm}) \\
\text { ref. [31] }\end{array}$ & $\begin{array}{c}\lambda_{c}(\mathrm{~cm}) \\
\text { (elliptical arcs) }\end{array}$ & $\begin{array}{c}\text { Relative error } \\
(\%)\end{array}$ \\
\hline 1 & TE & 3.394477 & 3.394426 & 0.0015 \\
5 & TE & 1.907950 & 1.907890 & 0.0030 \\
10 & TE & 1.397907 & 1.397804 & 0.0073 \\
20 & TE & 0.916070 & 0.915912 & 0.0170 \\
30 & TM & 0.775601 & 0.775505 & 0.0120 \\
50 & TM & 0.592145 & 0.592021 & 0.0200 \\
70 & TE & 0.494025 & 0.493878 & 0.0290 \\
90 & TM & 0.434155 & 0.433990 & 0.0380 \\
100 & TE & 0.416163 & 0.415935 & 0.0540 \\
\hline
\end{tabular}

this case, only circular arcs) BI-RME technique, is presented. In both cases, the circular contour has been divided into only ten segments. As can be seen in Table I, an important accuracy improvement is obtained with the new theory proposed for circular arcs.

The next canonical example considered has been an elliptical waveguide with major semiaxis $a=10.0 \mathrm{~mm}$ and eccentricity $e=0.5$ since results for the cutoff frequencies of such waveguides have been extensively reported in the literature. In order to apply the extended BI-RME technique proposed in this paper, the ellipse under consideration has been defined within a rectangular waveguide of dimensions $21 \mathrm{~mm}$ $\times 18 \mathrm{~mm}$, and has been segmented using 176 smaller elliptical arcs. Using this technique, the first 181 modes of the considered elliptical waveguide have been computed (100 TE modes and 81 TM solutions). Table II successfully compares the cutoff wavelengths for the first 100 modal solutions with results from [31], where a completely different approach for solving the modal problem was proposed. The total CPU time required to solve this example has been of $47 \mathrm{~s}$, which is rather well compared with the $167 \mathrm{~s}$ related to the method proposed in [31] and the $303 \mathrm{~s}$ (also reported in [31]) of a standard package for solving the well-known Mathieu functions. These last two CPU times have been obtained using an IBM RISC-6000 workstation.

Once the new theory proposed has been successfully validated, we considered a final example of great practical interest, i.e., the cross-shaped iris shown in Fig. 4. As already explained in Section I, this coupling iris is commonly used in circular waveguide dual-mode filters, which are widely used for space applications. Furthermore, most of the modern low-cost fabrication techniques of these irises, such as computer-controlled milling, spark eroding, electro-forming, or die casting, usually introduce rounded corners, as shown in Fig. 4. The accurate consideration of such a mechanization effect by the future CAD tools of dual-mode filters would extremely reduce the current

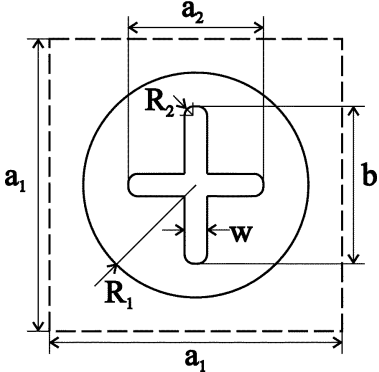

Fig. 4. Cross-shaped iris with rounded corners of different radius $\left(R_{2}=0,0.01,0.1,0.2,0.5\right.$ and $\left.1.0 \mathrm{~mm}\right)$. The other dimensions are $a_{2}=15.3 \mathrm{~mm}, b=17.3 \mathrm{~mm}, w=2 \mathrm{~mm}$, and $R_{1}=12.0 \mathrm{~mm}$. To solve this example, a square box of $a_{1}=25 \mathrm{~mm}$ has been used.

TABLE III

First CUTOFF WAVENUMBERS $\left(\mathrm{mm}^{-1}\right)$ AND COUPLING COEFFICIENTS OF THE CROSS-SHAPED IRIS With RIGHT-ANGLE CORNERS $\left(R_{2}=0 \mathrm{~mm}\right)$ SHOWN IN Fig. 4. The Results are Compared With Those Provided by [25]

\begin{tabular}{l|l|l}
\hline \multicolumn{1}{c|}{$\mathrm{TE}_{1}$} & \multicolumn{1}{c}{$\mathrm{TE}_{2}$} & \multicolumn{1}{c}{$\mathrm{TE}_{3}$} \\
$k_{c}=0.18762$ & $k_{c}=0.21136$ & $k_{c}=0.21305$ \\
\hline$E r r=0.021 \%$ & $E r r=0.03 \%$ & $E r r=0.01 \%$ \\
\hline \hline $0.32821\left(\mathrm{TE}_{11 c}\right)$ & $-0.21689\left(\mathrm{TE}_{21 s}\right)$ & $0.31893\left(\mathrm{TE}_{11 s}\right)$ \\
$0.32817^{*}$ & $-0.21687^{*}$ & $0.31892^{*}$ \\
\hline$-0.37204\left(\mathrm{TM}_{11 s}\right)$ & $-0.04555\left(\mathrm{TE}_{01}\right)$ & $0.36430\left(\mathrm{TM}_{11 c}\right)$ \\
$-0.37234^{*}$ & $-0.04556^{*}$ & $0.36469^{*}$ \\
\hline$-0.08293\left(\mathrm{TE}_{31 c}\right)$ & $-0.35096\left(\mathrm{TM}_{21 c}\right)$ & $0.06622\left(\mathrm{TE}_{31 s}\right)$ \\
$-0.08490^{*}$ & $-0.35523^{*}$ & $0.06829^{*}$ \\
\hline $0.16007\left(\mathrm{TE}_{21 c}\right)$ & $0.01694\left(\mathrm{TE}_{41 s}\right)$ & $0.20223\left(\mathrm{TE}_{12 s}\right)$ \\
$0.16012^{*}$ & $0.01688^{*}$ & $0.20216^{*}$ \\
\hline $0.17295\left(\mathrm{TM}_{31 s}\right)$ & $-0.20719\left(\mathrm{TE}_{22 s}\right)$ & $0.14331\left(\mathrm{TM}_{31 c}\right)$ \\
$0.17080^{*}$ & $-0.20726^{*}$ & $0.14110^{*}$ \\
\hline
\end{tabular}

fabrication costs and development times of such complex devices.

First, for verification purposes, we have considered a crossshaped iris with straight-angle corners since numerical results for this simpler case can be found in the technical literature [25]. The structure under study can be seen in Fig. 4, where $R_{2}$ has been obviously chosen to be equal to $0 \mathrm{~mm}$ (right-angle corners), $a_{2}=15.3 \mathrm{~mm}, b=17.3 \mathrm{~mm}$, and $w=2 \mathrm{~mm}$. To apply the BI-RME method, a square surrounding box $\left(a_{1}=25 \mathrm{~mm}\right)$ has been chosen. Table III reports the cutoff wavenumbers (in $\mathrm{mm}^{-1}$ ) provided by our BI-RME implementation for the first three TE modes of the described iris, as well as the relative error (Err) between such results and those collected in [25]. Table III also provides the values of the coupling coefficients among the computed modes of the cited cross-shaped iris and the first TE and TM modes of a standard circular waveguide of radius $R_{1}=12.0 \mathrm{~mm}$, also shown in Fig. 4 . Our results are those next to the parenthesis enclosing the standard circular 
TABLE IV

Cutoff WaVenumbers of the Three Lowest Order Modes of THE Cross-Shaped IRIS With Rounded Corners $\left(R_{2}=0.01,0.1,0.2,0.5\right.$, AND $\left.1.0 \mathrm{~mm}\right)$ SHOWN IN FIG. 4

\begin{tabular}{c||c|c|c|c|c}
\hline Mode & $k_{c}\left(\mathrm{~mm}^{-1}\right)$ & $k_{c}\left(\mathrm{~mm}^{-1}\right)$ & $k_{c}\left(\mathrm{~mm}^{-1}\right)$ & $k_{c}\left(\mathrm{~mm}^{-1}\right)$ & $k_{c}\left(\mathrm{~mm}^{-1}\right)$ \\
$(\mathrm{TE} / \mathrm{TM})$ & $R_{2}=0.01 \mathrm{~mm}$ & $R_{2}=0.1 \mathrm{~mm}$ & $R_{2}=0.2 \mathrm{~mm}$ & $R_{2}=0.5 \mathrm{~mm}$ & $R_{2}=1.0 \mathrm{~mm}$ \\
\hline \hline $\mathrm{TE}_{1}$ & 0.18762 & 0.18766 & 0.18781 & 0.18882 & 0.19253 \\
$\mathrm{TE}_{2}$ & 0.21136 & 0.21142 & 0.21160 & 0.21289 & 0.21761 \\
$\mathrm{TE}_{3}$ & 0.21305 & 0.21311 & 0.21330 & 0.21461 & 0.21940 \\
\hline
\end{tabular}

waveguide modes considered, whereas those marked with an asterisk have been obtained from [25]. As can be observed, an excellent agreement between both results is obtained.

Next, the new theory developed in Section II has been used to solve the modal chart of the previous cross-shaped iris, but now considering different curvature radius ( $R_{2}=0.01,0.1,0.2,0.5$ and $1.0 \mathrm{~mm}$ ) for the rounded corners shown in Fig. 4. The evolution of the cutoff wavenumbers (in $\mathrm{mm}^{-1}$ ) for the first three TE modes of the perturbed iris, in terms of the different radius values chosen for the rounded corners, is offered in Table IV. As can be seen in this table, even for small values of the rounded corners radius $\left(R_{2}=1.0 \mathrm{~mm}\right)$, the cutoff wavenumbers of the very low-order modes begin to be considerably modified (relative differences approximately $3 \%$ ). Therefore, the inclusion of these effects in the modern CAD tools is revealed as being rather necessary, especially for machined components to operate in the higher microwave and millimeter-wave bands. For the example we have just studied, the arbitrarily shaped contour has been divided into 164 arcs (straight and circular ones), and the first 16 modes (15 TE and $1 \mathrm{TM}$ ) of the strongly perturbed iris have been computed, thus requiring a total CPU effort of only $30 \mathrm{~s}$.

\section{B. Analysis and Design of Complex Passive Waveguide Devices}

Once the novel theory proposed has been previously validated with several benchmark tests, its direct application to the analysis and design of modern complex passive waveguide devices is faced. For that purpose, we have integrated the new BI-RME extended technique proposed in this paper within a CAD software package based on the integral-equation method fully described in [32]. As indicated in [32], this efficient full-wave analysis method requires the knowledge of the modal chart related to all the waveguides included in the devices under consideration. In order to solve the modal chart of the arbitrarily shaped waveguides that can be present in modern passive waveguide devices, we have made use of the efficient and accurate modal analysis tool developed in the context of this study.

Before using the new CAD software package developed for the design of novel components, we have tested its accuracy and efficiency with a complex passive waveguide device involving circular and elliptical waveguides, which has been recently reported in the literature. This complex device is a four-pole dual-mode filter successfully designed in [8], which is of great

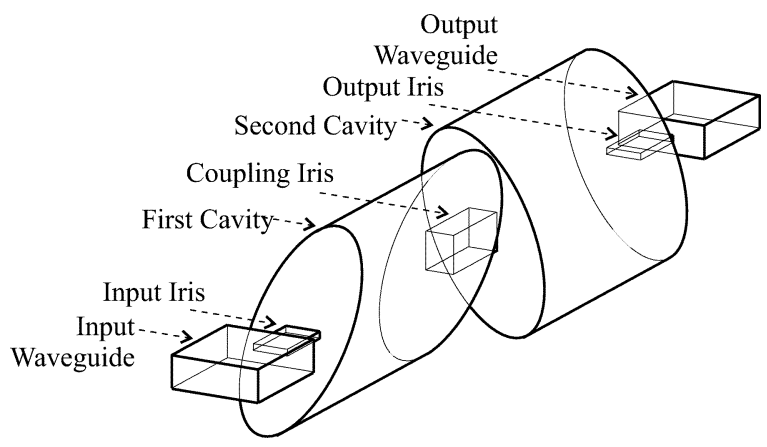

Fig. 5. Four-pole dual-mode filter with elliptical waveguide resonators in standard WR-75 rectangular waveguides ( $a=19.050 \mathrm{~mm}, b=9.525 \mathrm{~mm})$. The dimensions are: input iris $(9.91 \mathrm{~mm} \times 2.0 \mathrm{~mm})$ of length $2.0 \mathrm{~mm}$, first elliptical cavity (major semiaxis of $11.0 \mathrm{~mm}$, minor semiaxis of $10.50 \mathrm{~mm}$, and rotation angle of $\left.81.46^{\circ}\right)$ of length $16.62 \mathrm{~mm}$, coupling central iris $(3.5 \mathrm{~mm} \times$ $4.98 \mathrm{~mm}$ ) of length $1.0 \mathrm{~mm}$, second elliptical cavity (major semiaxis of $11.0 \mathrm{~mm}$, minor semiaxis of $10.50 \mathrm{~mm}$, and rotation angle of $98.54^{\circ}$ ) of length $16.62 \mathrm{~mm}$, and output iris $(9.91 \mathrm{~mm} \times 2.0 \mathrm{~mm})$ of length $2.0 \mathrm{~mm}$.

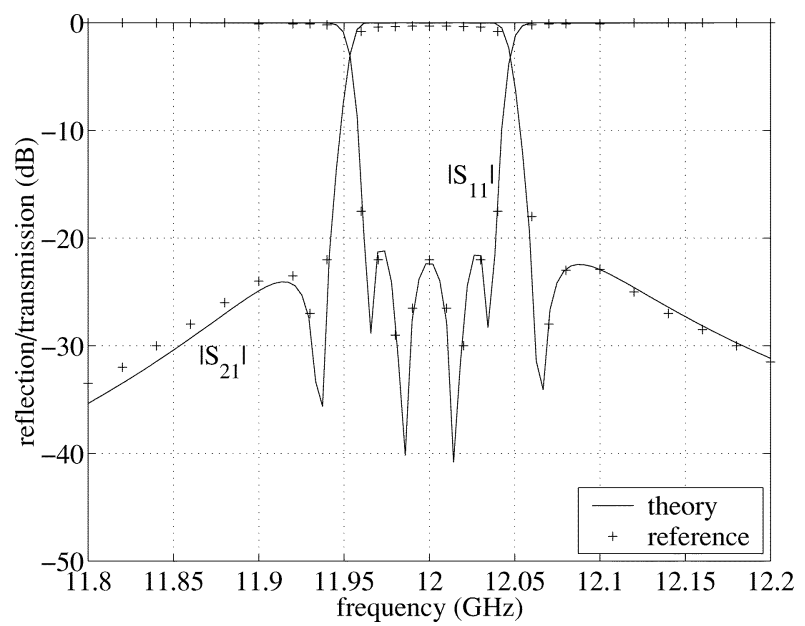

Fig. 6. Magnitude of the reflection $\left(S_{11}\right)$ and transmission $\left(S_{21}\right)$ coefficients of the four-pole dual-mode filter with elliptical cavities shown in Fig. 5. The authors' results are denoted by the solid line. Crosses denote the numerical results collected from [8].

use for narrow-band applications. As can be seen in Fig. 5, this original structure is composed of two elliptical cavities coupled through a rectangular iris, which allows the avoidance of the typical presence of tuning and coupling elements in these types of devices. The geometric parameters of this structure can also be found in Fig. 5. The simulated reflection and transmission coefficients of this compact device are compared in Fig. 6 


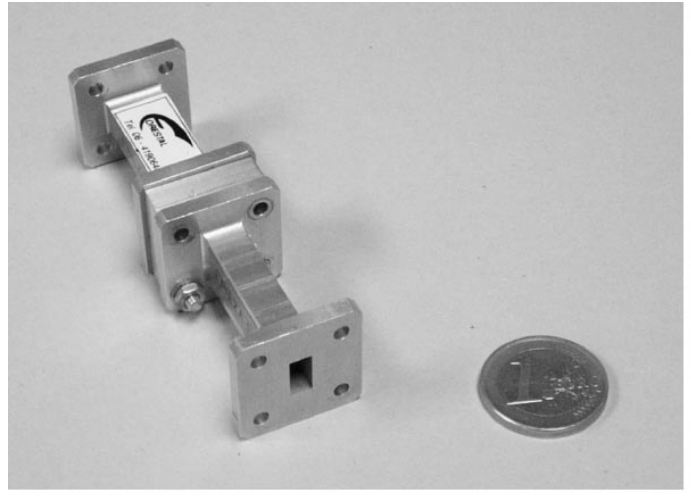

(a)

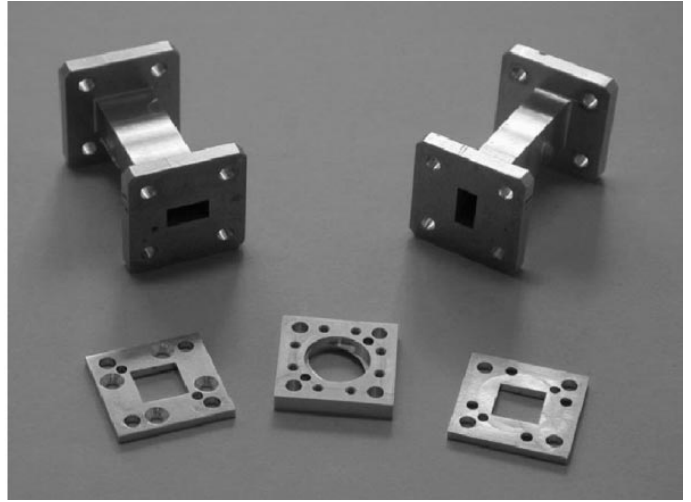

(b)

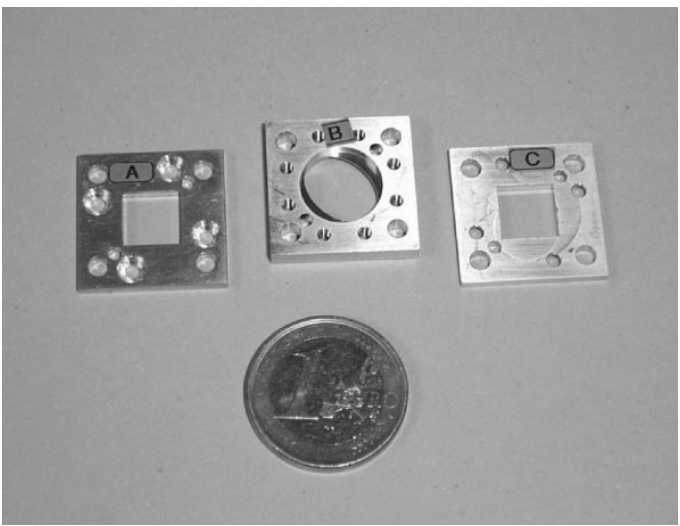

(c)

Fig. 7. Photographs of the manufactured $90^{\circ}$ twist component prototype. (a) Complete view of the twist manufactured in standard WR-34 rectangular waveguides ( $a=8.636 \mathrm{~mm}, b=4.318 \mathrm{~mm}$ ). (b) General view of all the waveguide pieces that integrate the component. (c) More detailed view of the internal pieces of the device, i.e., the two square waveguides of size $8.636 \mathrm{~mm}$ and length $1.80 \mathrm{~mm}$, and a central piece with two circular waveguides of radius $6.10 \mathrm{~mm}$ and length $1.60 \mathrm{~mm}$ and an inner elliptical iris (major semiaxis of $6.0 \mathrm{~mm}$, minor semiaxis of $3.90 \mathrm{~mm}$ and rotation angle of $45.0^{\circ}$ ) of length $0.30 \mathrm{~mm}$

with the numerical results provided by [8]. A very good agreement between both results can be observed, even though a slight difference is noticed in the lower rejected frequency band. However, the experimental results of a manufactured prototype in such a low-frequency band, also reported in [8], fit better with our simulated results. To reach our convergent results, seven accessible modes, 20 basis functions, and 400 kernel terms in the integral equations were required. Due to the great complexity of this device, the complete simulation of its electrical response has taken a CPU effort of $7.2 \mathrm{~s}$ per frequency point.

Finally, making use of the CAD software package produced, we have designed a new $90^{\circ}$ twist component for $K$-band applications. Up to now, $90^{\circ}$ twist components have been designed using L-shaped rectangular waveguides (see, for instance, [33]). Here, we propose an alternative compact geometry for such components based on a soft rotation of the $E$-field through successive square, circular, and elliptical waveguides. A prototype of such a device, which is intended to operate at $26.3 \mathrm{GHz}$ with a wide bandwidth of approximately $2 \mathrm{GHz}$, has been designed and manufactured. Photographs of this prototype, as well as of their integrating pieces, are displayed in Fig. 7, where the geometric dimensions of all such pieces are also collected. The simulated scattering parameters of this novel two-port device are shown in Fig. 8, where they are successfully compared with authors' measurements. During the design procedure of the twist

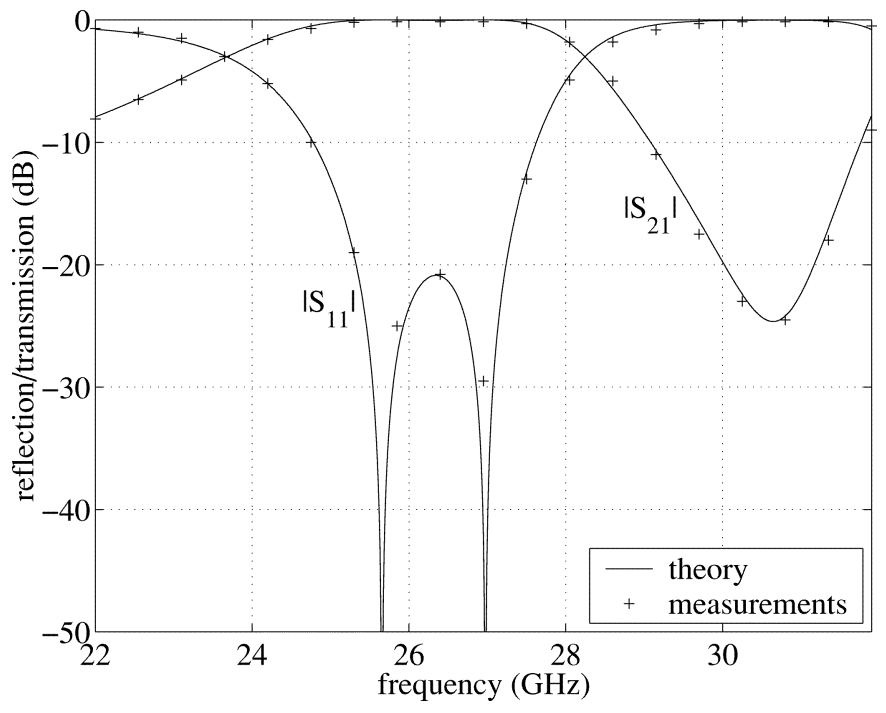

Fig. 8. Magnitude of the reflection $\left(S_{11}\right)$ and transmission $\left(S_{21}\right)$ coefficients of the 90-twist component for $K$-band applications shown in Fig. 7. Solid line denotes the authors' results. Crosses denoted the authors' measurements.

component, convergent simulation results were obtained using 20 accessible modes, 50 basis functions, and 400 kernel terms in the integral equations. These simulating parameters only involved a total computational effort of $0.54 \mathrm{~s}$ per frequency point, which is appropriate for design purposes. 


\section{CONCLUSIONS}

Arbitrarily shaped waveguides, composed of circular and elliptical arcs, are increasingly used in modern passive waveguide components. This paper has described an efficient way for the very accurate consideration of such types of arcs within the classical well-known BI-RME formulation, which, up to now, has always been implemented considering only straight segments for defining the arbitrary profiles. The new theory proposed has been extensively verified through several application examples of great practical interest. First, the modal chart of canonical circular and elliptical waveguides have been successfully computed. Next, the new extended method has been applied to the accurate modal analysis of widely used cross-shaped irises, where completely new results considering the presence of rounded corners due to undesirable mechanization effects have also been offered. Finally, the new modal analysis technique developed has been used together with a CAD software package for advanced analysis and design purposes. After validating this powerful CAD tool with the analysis of a complex waveguide device involving circular and elliptical waveguides, a novel twist component for $K$-band applications has been successfully designed, manufactured, and measured. CPU times for the previous examples have been included to prove the good numerical efficiency of the new modal analysis tool developed.

\section{APPENDIX I}

\section{ANALYTICAL EXPRESSIONS FOR SINGULAR INTEGRALS}

Here, the analytical expressions for all of the singular integrals appearing in Section II are collected.

The integral of a parabolic spline multiplied by a logarithmic singular term has the following analytical solution:

$$
\begin{aligned}
\int_{-\frac{1}{2}}^{\frac{1}{2}}\left(a \xi^{\prime 2}+b \xi^{\prime}+c\right) \ln \left[\varphi^{2}\left(\xi-\xi^{\prime}\right)^{2}\right] d \xi^{\prime} \\
=\left\{\frac{1}{12}\left[8 a \xi^{3}+12 b \xi^{2}+24 c \xi+a-3(b-4 c)\right] \ln |2 \xi+1|\right. \\
\quad-\frac{1}{12}\left[8 a \xi^{3}+12 b \xi^{2}+24 c \xi-a-3(b+4 c)\right] \ln |2 \xi-1| \\
\left.+\left(\frac{a}{6}+2 c\right) \ln \left|\frac{\varphi}{2}\right|-\frac{1}{18}\left(12 a \xi^{2}+18 b \xi+a+36 c\right)\right\} .
\end{aligned}
$$

For the TE case and circular/elliptic arcs, the unitary tangent vector to circular and elliptical arcs is pre- and post-multiplying the solenoidal dyadic function $\overline{\mathbf{G}}_{s t}$, thus giving rise to the following two singular integrals whose analytical solutions are also offered:

$$
\begin{aligned}
& \int_{-\frac{1}{2}}^{\frac{1}{2}}\left(a \xi^{2}+b \xi^{\prime}+c\right) \sin \left(\varphi \xi^{\prime}+\varphi_{0}\right) \ln \left[\varphi^{2}\left(\xi-\xi^{\prime}\right)^{2}\right] d \xi^{\prime} \\
& \quad=\left(a I_{2}^{s}+b I_{1}^{s}+c I_{0}^{s}\right) \cos \left(\varphi_{0}\right)+\left(a I_{2}^{c}+b I_{1}^{c}+c I_{0}^{c}\right) \sin \left(\varphi_{0}\right)
\end{aligned}
$$

$$
\begin{aligned}
& \int_{-\frac{1}{2}}^{\frac{1}{2}}\left(a \xi^{\prime 2}+b \xi^{\prime}+c\right) \cos \left(\varphi \xi^{\prime}+\varphi_{0}\right) \ln \left[\varphi^{2}\left(\xi-\xi^{\prime}\right)^{2}\right] d \xi^{\prime} \\
& \quad=\left(a I_{2}^{c}+b I_{1}^{c}+c I_{0}^{c}\right) \cos \left(\varphi_{0}\right)-\left(a I_{2}^{s}+b I_{1}^{s}+c I_{0}^{s}\right) \sin \left(\varphi_{0}\right)
\end{aligned}
$$

The integrals $I_{0}^{s}, I_{1}^{s}, I_{2}^{s}, I_{0}^{c}, I_{1}^{c}$, and $I_{2}^{c}$, which have been introduced in the previous expressions, are defined as follows:

$$
\begin{aligned}
I_{0}^{s}=\frac{1}{\varphi} \int_{-\frac{\varphi}{2}}^{\frac{\varphi}{2}} \sin (x) \ln (k-x)^{2} d x \\
=\frac{2}{\varphi}\left\{\cos \left(\frac{\varphi}{2}\right) \ln \left|\frac{\varphi}{\frac{\varphi}{\varphi}+k}\right|\right. \\
\quad+\cos k\left[\operatorname{Ci}\left(k-\frac{\varphi}{2}\right)-\operatorname{Ci}\left(k+\frac{\varphi}{2}\right)\right] \\
\left.\quad+\sin k\left[\operatorname{Si}\left(k-\frac{\varphi}{2}\right)-\operatorname{Si}\left(k+\frac{\varphi}{2}\right)\right]\right\}
\end{aligned}
$$

$$
\begin{aligned}
I_{1}^{s}=\frac{1}{\varphi^{2}} \int_{-\frac{\varphi}{2}}^{\frac{\varphi}{2}} x \sin (x) \ln (k-x)^{2} d x \\
=\frac{2}{\varphi^{2}}\left\{\left[\sin \left(\frac{\varphi}{2}\right)-\frac{\varphi}{2} \cos \left(\frac{\varphi}{2}\right)\right] \ln \left|\left(\frac{\varphi}{2}-k\right)\left(\frac{\varphi}{2}+k\right)\right|\right. \\
+(k \cos k-\sin k)\left[\operatorname{Ci}\left(k-\frac{\varphi}{2}\right)-\mathrm{Ci}\left(k+\frac{\varphi}{2}\right)\right] \\
\left.+(k \sin k+\cos k)\left[\operatorname{Si}\left(k-\frac{\varphi}{2}\right)-\operatorname{Si}\left(k+\frac{\varphi}{2}\right)\right]\right\} \\
+2 \sin \left(\frac{\varphi}{2}\right)
\end{aligned}
$$

$$
\begin{aligned}
I_{2}^{s}=\frac{1}{\varphi^{3}} \int_{-\frac{\varphi}{2}}^{\frac{\varphi}{2}} x^{2} \sin (x) \ln (k-x)^{2} d x \\
=\frac{2}{\varphi^{3}}\left\{\left[\varphi \sin \left(\frac{\varphi}{2}\right)-\left(\frac{\varphi^{2}}{4}-2\right) \cos \left(\frac{\varphi}{2}\right)\right] \ln \left|\frac{\frac{\varphi}{2}-k}{\frac{\varphi}{2}+k}\right|\right. \\
+\left[\left(k^{2}-2\right) \cos k-2 k \sin k\right] \\
\quad \times\left[\operatorname{Ci}\left(k-\frac{\varphi}{2}\right)-\operatorname{Ci}\left(k+\frac{\varphi}{2}\right)\right] \\
+\quad\left[\left(k^{2}-2\right) \sin k+2 k \cos k\right] \\
\left.\quad \times\left[\operatorname{Si}\left(k-\frac{\varphi}{2}\right)-\operatorname{Si}\left(k+\frac{\varphi}{2}\right)\right]\right\} \\
+2 k \sin \left(\frac{\varphi}{2}\right) \\
I_{0}^{c}=\frac{1}{\varphi} \int_{-\frac{\varphi}{2}}^{\frac{\varphi}{2}} \cos (x) \ln (k-x)^{2} d x \\
=\frac{2}{\varphi}\left\{\sin \left(\frac{\varphi}{2}\right) \ln \left|\left(\frac{\varphi}{2}+k\right)\left(\frac{\varphi}{2}-k\right)\right|\right. \\
\quad-\sin k\left[\operatorname{Ci}\left(k-\frac{\varphi}{2}\right)-\operatorname{Ci}\left(k+\frac{\varphi}{2}\right)\right] \\
\left.\quad+\cos k\left[\operatorname{Si}\left(k-\frac{\varphi}{2}\right)-\operatorname{Si}\left(k+\frac{\varphi}{2}\right)\right]\right\}
\end{aligned}
$$




$$
\begin{aligned}
& I_{1}^{c}=\frac{1}{\varphi^{2}} \int_{-\frac{\varphi}{2}}^{\frac{\varphi}{2}} x \cos (x) \ln (k-x)^{2} d x \\
&=\frac{2}{\varphi^{2}}\left\{\left[\cos \left(\frac{\varphi}{2}\right)+\frac{\varphi}{2} \sin \left(\frac{\varphi}{2}\right)\right] \ln \left|\frac{\varphi}{\frac{\varphi}{\varphi}-k}\right|\right. \\
&-(k \sin k+\cos k) \\
& \times\left[\operatorname{Ci}\left(k-\frac{\varphi}{2}\right)-\mathrm{Ci}\left(k+\frac{\varphi}{2}\right)\right] \\
&+(k \cos k-\sin k) \\
&\left.\times\left[\operatorname{Si}\left(k-\frac{\varphi}{2}\right)-\operatorname{Si}\left(k+\frac{\varphi}{2}\right)\right]\right\} \\
& I_{2}^{c}=\frac{1}{\varphi^{3}} \int_{-\frac{\varphi}{2}}^{\frac{\varphi}{2}} x^{2} \cos (x) \ln (k-x)^{2} d x \\
&=\frac{2}{\varphi^{3}}\left\{\left[\varphi \cos \left(\frac{\varphi}{2}\right)+\left(\frac{\varphi^{2}}{4}-2\right) \sin \left(\frac{\varphi}{2}\right)\right]\right. \\
& \times \ln \left|\left(\frac{\varphi}{2}-k\right)\left(\frac{\varphi}{2}+k\right)\right| \\
&+\varphi \cos \left(\frac{\varphi}{2}\right)-6 \sin \left(\frac{\varphi}{2}\right) \\
&-\left[\left(k^{2}-2\right) \sin k+2 k \cos k\right] \\
& \times\left[\operatorname{Ci}\left(k-\frac{\varphi}{2}\right)-\operatorname{Ci}\left(k+\frac{\varphi}{2}\right)\right] \\
&+\left[\left(k^{2}-2\right) \cos k-2 k \sin k\right] \\
&\left.\times\left[\operatorname{Si}\left(k-\frac{\varphi}{2}\right)-\operatorname{Si}\left(k+\frac{\varphi}{2}\right)\right]\right\} .
\end{aligned}
$$

In the expressions collected in (40), $k={ }_{\varphi \xi}$ and

$$
\begin{aligned}
& \operatorname{Si}(x)=\int_{0}^{x} \frac{\sin (\tau)}{\tau} d \tau=\sum_{n=0}^{\infty} \frac{(-1)^{n} x^{2 n+1}}{(2 n+1)(2 n+1) !} \\
& \operatorname{Ci}(x)=\int_{\infty}^{x} \frac{\cos (\tau)}{\tau} d \tau=\gamma+\ln (x)+\sum_{n=1}^{\infty} \frac{(-1)^{n} x^{2 n}}{(2 n)(2 n) !}
\end{aligned}
$$

where $\gamma$ is the Euler's constant.

\section{REFERENCES}

[1] X.-P. Liang, K. A. Zaki, and A. E. Atia, "Dual mode coupling by square corner cut in resonators and filters," IEEE Trans. Microwave Theory Tech., vol. 40, pp. 2294-2302, Dec. 1992.

[2] M. Guglielmi, R. C. Molina, and A. Alvarez, "Dual-mode circular waveguide filters without tuning screws," IEEE Microwave Guided Wave Lett., vol. 2, pp. 457-458, Nov. 1992.

[3] P. Couffignal, H. Baudrand, and B. Théron, "A new rigorous method for the determination of iris dimensions in dual-mode cavity filters," IEEE Trans. Microwave Theory Tech., vol. 42, pp. 1314-1320, July 1994.

[4] V. E. Boria, G. Gerini, and M. Guglielmi, "Computer aided design of reentrant coaxial filter including coaxial excitation," in IEEE MTT-S Int. Microwave Symp. Dig., vol. 3, June 1999, pp. 1131-1134.

[5] P. Soto, V. E. Boria, J. M. Catalá, N. Chouaib, M. Guglielmi, and B. Gimeno, "Analysis, design and experimental verification of microwave filters for safety issues in open-ended waveguide systems," IEEE Trans. Microwave Theory Tech., vol. 48, pp. 2133-2140, Nov. 2000.

[6] S. Cogollos, V. E. Boria, P. Soto, B. Gimeno, and M. Guglielmi, "Efficient CAD tool for inductively coupled rectangular waveguide filters with rounded corners," in Proc. 31st Eur. Microwave Conf., vol. 1, Sept. 2001, pp. 315-318.
[7] R. Kühne and J. Marquardt, "Mutual coupling of open-ended waveguides with arbitrary cross-sections located in an infinite ground plane," in Proc. 30th Eur. Microwave Conf., vol. 2, Oct. 2000, pp. 357-360.

[8] L. Accatino, G. Bertin, and M. Mongiardo, "Elliptical cavity resonators for dual-mode narrow-band filters," IEEE Trans. Microwave Theory Tech., vol. 45, pp. 2393-2401, Dec. 1997.

[9] —_, "An elliptical cavity for triple-mode filters," in IEEE MTT-S Int. Microwave Symp. Dig., vol. 3, June 1999, pp. 1037-1040.

[10] L. A. G. Bertin, B. Piovano, and M. Mongiardo, "Full-wave design and optimization of circular waveguide polarizers with elliptical irises," IEEE Trans. Microwave Theory Tech., vol. 50, pp. 1077-1083, Apr. 2002.

[11] G. L. James, "Propagation and radiation from partially filled elliptical waveguide," Proc. Inst. Elect. Eng., pt. H, vol. 136, pp. 195-201, June 1989.

[12] F. A. Alharganm and S. R. Judah, "Tables of normalized cutoff wavenumbers of elliptical cross section resonators," IEEE Trans. Microwave Theory Tech., vol. 42, pp. 332-338, Feb. 1994.

[13] P. J. B. Clarricoats and A. D. Olver, Corrugated Horns for Microwave Antennas. London, U.K.: Peregrinus, 1984.

[14] H. H. Meinke, K. P. Lange, and J. F. Ruger, "TE- and TM waves in waveguides of very general cross section," Proc. IEEE, pp. 1436-1443, Nov. 1963

[15] J. B. Davies, "Review of methods for numerical solution of the hollowwaveguide problem," Proc. Inst. Elect. Eng., vol. 119, pp. 33-37, Jan. 1972.

[16] F. L. Ng, "Tabulation of methods for the numerical solution of the hollow waveguide problem," IEEE Trans. Microwave Theory Tech., vol. MTT-22, pp. 322-329, Mar. 1974

[17] J. Mazumdar, "A method for the study of TE and TM modes in waveguides of very general cross section," IEEE Trans. Microwave Theory Tech., vol. 28, pp. 991-995, Sept. 1980.

[18] L. Gruner, "Characteristics of crossed rectangular coaxial structures," IEEE Trans. Microwave Theory Tech., vol. MTT-28, pp. 622-627, June 1980.

[19] N. P. Malakshinov and A. S. Smagin, "Investigation of arbitrarily shaped regular waveguides by the method of auxiliary sources," Radio Eng. Elect. Phys., vol. 27, pp. 56-60, June 1982.

[20] P. Saguet and E. Pic, "Le maillage rectangulaire et le changement de maille dans la methode TLM en deux dimensions," Electron. Lett., vol. 17, no. 7, pp. 277-279, Apr. 1981.

[21] M. Israel and R. Miniowitz "An efficient finite element method for nonconvex waveguide based on Hermitian polynomials," IEEE Trans. Microwave Theory Tech., vol. MTT-35, pp. 1019-1026, Nov. 1987.

[22] A. S. Omar and K. F. Schünemann, "Application of the generalized spectral-domain technique to the analysis of rectangular waveguides with rectangular and circular metal inserts," IEEE Trans. Microwave Theory Tech., vol. 39, pp. 944-952, June 1991.

[23] W. L. Schroeder and M. Guglielmi, "A contour-based approach to the multimode network representation of waveguide transitions," IEEE Trans. Microwave Theory Tech., vol. 46, pp. 411-419, Apr. 1998.

[24] G. Conciauro, M. Bressan, and C. Zuffada, "Waveguide modes via an integral equation leading to a linear matrix eigenvalue problem," IEEE Trans. Microwave Theory Tech., vol. MTT-32, pp. 1495-1504, Nov. 1984.

[25] P. Arcioni, "Fast evaluation of modal coupling coefficients of waveguide step discontinuities," IEEE Microwave Guided Wave Lett., vol. 6, pp. 232-234, June 1996.

[26] M. Bozzi, G. Conciauro, and L. Perregrini, "On the evaluation of modal coupling coefficients by contour integrals," IEEE Trans. Microwave Theory Tech., vol. 50, pp. 1853-1855, July 2002.

[27] G. Conciauro, M. Guglielmi, and R. Sorrentino, Advanced Modal Analysis-CAD Techniques for Waveguide Components and Filters. Chichester, U.K.: Wiley, 2000.

[28] P. Arcioni, M. Bressan, and G. Conciauro, "Wideband analysis of planar waveguide circuits," Alta Freq., vol. 57, no. 5, pp. 217-226, June 1988.

[29] P. K. Kythe and P. Puri, Computational Methods for Lineal Integral Equations. Boston, MA: Birkhäuser, 2002.

[30] J. J. H. Wang, Generalized Moment Methods in Electromagnetics: Formulation and Computer Solution of Integral Equations. New York: Wiley, 1991.

[31] B. Gimeno and M. Guglielmi, "Full wave network representation for rectangular, circular, and elliptical to elliptical waveguide junctions," IEEE Trans. Microwave Theory Tech., vol. 45, pp. 376-384, Mar. 1997.

[32] G. Gerini, M. Guglielmi, and G. Lastoria, "Efficient integral formulations for admittance or impedance representation of planar waveguide junctions," in IEEE MTT-S Int. Microwave Symp. Dig., vol. 3, June 1998, pp. $1747-1750$.

[33] H. F. Lenzing and M. J. Gans, "Machined waveguide twist," IEEE Trans. Microwave Theory Tech., vol. 38, pp. 942-944, July 1990. 


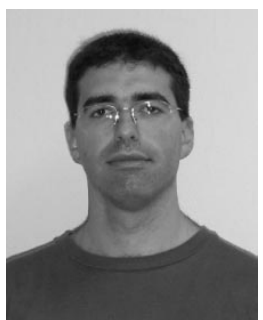

Santiago Cogollos was born in Valencia, Spain, on January 15, 1972. He received the Ingeniero de Telecomunicación degree and the Doctor Ingeniero de Telecomunicación degree from the Universidad Politécnica de Valencia, Valencia, Spain, in 1996 and 2002, respectively.

In 2000, he joined the Departamento de Comunicaciones, Universidad Politécnica de Valencia, where he was an Assistant Lecturer from 2000 to 2001, a Lecturer from 2001 to 2002, and became an Associate Professor in 2002. He has collaborated with the European Space Research and Technology Centre (ESTEC), European Space Agency (ESA), in the development of modal analysis tools for payload systems in satellites. His current research interests include numerical methods for the analysis of waveguide structures and design of waveguide components for space applications.

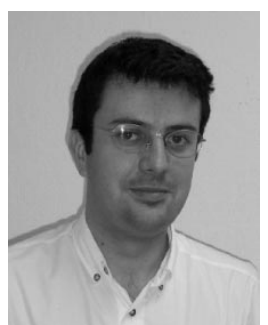

Pablo Soto was born in Cartagena, Spain, on August 10, 1975. He received the Ingeniero de Telecomunicación degree from the Universidad Politécnica de Valencia, Valencia, Spain, in 1999.

In 2000, he joined the European Space Research and Technology Centre, European Space Agency (ESTEC-ESA), Noordwijk, The Netherlands as a European Research Fellow. In October 2000, he joined the Departamento de Comunicaciones, Universidad Politécnica de Valencia, where he is currently an Associate Lecturer. His current research activities are focused on the development of software tools for the analysis and optimized design of passive waveguide structures.

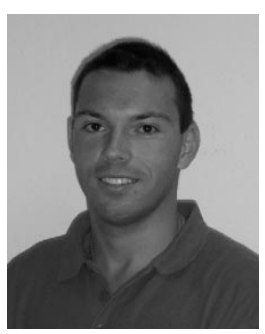

Stephan Marini was born in Cagli, Italy, on January 3, 1976. He received the Laurea degree in electronics engineering from the University of Perugia, Perugia, Italy, in 2001, and is currently working toward the $\mathrm{Ph} . \mathrm{D}$. degree in telecommunications at the Universidad Politécnica de Valencia, Valencia, Spain.

In June 2001 he joined the Departamento de Comunicaciones, Universidad Politécnica de Valencia. His current research interests include numerical methods for the analysis of arbitrarily shaped waveguide and scattering structures.

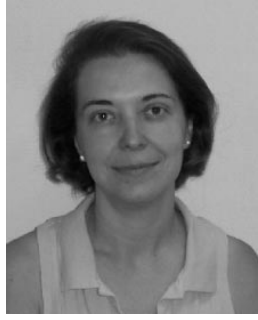

Ana Vidal was born in Valencia, Spain, in 1970. She received the Telecommunications Engineering degree from the Universidad Politecnica de Valencia, Valencia, Spain, in 1993. She spent one year with the University of Strathclyde, Glasgow, U.K., in 1993 under an international exchange program.

She worked with the Spanish electrical carrier Iberdrola during vacation. She was also a Trainee involved in broad-band communications development in the main research center of Telecom Portugal. She was then a Research Assistant with the Universidad Politecnica de Valencia. She was with the European Space Agency for two years as a Research Trainee, where her main activity was the study and implementation of software for synthetic aperture radar (SAR) image processing. In 1996, she returned to the Universidad Politecnica de Valencia, where she held several lecturing positions. Since 2001, she has been an Associate Professor with the Universidad Politecnica de Valencia. Her current interests are SAR data processing, SAR speckle noise reduction, and numerical methods for microwave structures analysis including the wavelet transform.

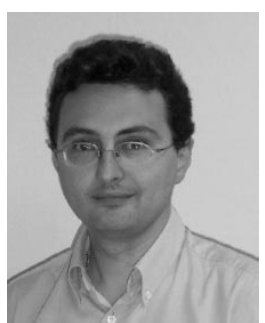

Vicente E. Boria (S'91-A'99-SM'02) was born in Valencia, Spain, on May 18, 1970. He received the Ingeniero de Telecomunicación degree (with first-class honors) and Doctor Ingeniero de Telecomunicación degree from the Universidad Politécnica de Valencia, Valencia, Spain, in 1993 and 1997, respectively.

In 1993, he joined the Departamento de Comunicaciones, Universidad Politécnica de Valencia, where he was an Assistant Lecturer (1993-1995), a Lecturer (1996-1997), and then became an Associate Professor in 1998. In 1995 and 1996, he held a Spanish Trainee position with the European Space Research and Technology Centre (ESTEC), European Space Agency (ESA), Noordwijk, The Netherlands, where he was involved in the area of electromagnetic analysis and design of waveguide devices. His current research interests include numerical methods for the analysis of waveguide and scattering structures, automated design of waveguide components, radiating systems, and measurement techniques. He has authored or coauthored over 20 papers in refereed international technical journals and over 70 papers in international conference proceedings in his areas of research interest.

Dr. Boria is a member of the IEEE Microwave Theory and Techniques Society (IEEE MTT-S) and the IEEE Antennas and Propagation Society (IEEE AP-S). Since 2003, he has been a member of the Technical Committee of the IEEE-MTT-S International Microwave Symposium (IMS) and of the European Microwave Conference. He was the recipient of the 1993 Spanish Ministerio de Educación y Ciencia, which is the First National Prize of Telecommunication Engineering Studies for his outstanding student record. He was also the recipient of the 2001 Social Council of the Universidad Politécnica de Valencia, which is the First Research Prize for his outstanding activity during 1995-2000.

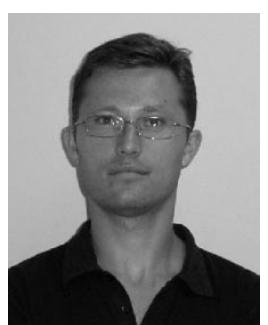

Hector Esteban (S'94-A'99) was born in Alicante, Spain, on May 12, 1972. He received the Telecommunications Engineering degree from the Universidad Politécnica de Valencia (UPV), Valencia, Spain, in 1996, and is currently working toward the Ph.D. degree at UPV. His doctoral research concerns the use of hybrid spectral and numerical techniques for the analysis of arbitrarily shaped $H$-plane devices in rectangular waveguides.

From 1994 to 1996, he was with the Communications Department, UPV, where he was involved in the development of spectral techniques for the electromagnetic characterization of land vegetation. He has collaborated with the Joint Research Centre, European Commission, Ispra, Italy, in the development of electromagnetic models for multiple tree trunks above a tilted ground plane. In 1997, he was with the European Topic Centre on Soil [European Environment Agency (ESA)], where he developed a GIS-integrated database for the assessment of European desertification. In 1998, he rejoined the Communications Department, UPV, as an Associate Professor. He has authored or coauthored eight papers in refereed international technical journals and over 30 papers in international conference proceedings in his areas of research interest. His research interests include methods for the full-wave analysis of open-space and guided multiple scattering problems, CAD of microwave devices, electromagnetic characterization of dielectric and magnetic bodies, and the acceleration of electromagnetic analysis methods using the wavelets. 


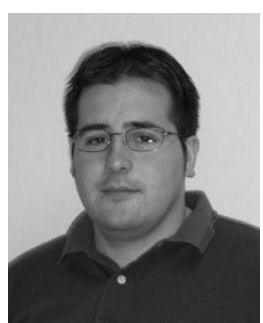

Jose V. Morro was born in Segorbe, Castellón, Spain, on April 3, 1978. He received the Telecommunications Engineering degree from the Universidad Politécnica de Valencia (UPV), Valencia, Spain, in 2001, and is currently working toward the Ph.D. degree at UPV.

Since 2001, he has been a Fellow Researcher with UPV. His current research interests include CAD design of microwave devices and the acceleration of electromagnetic analysis using wavelets.

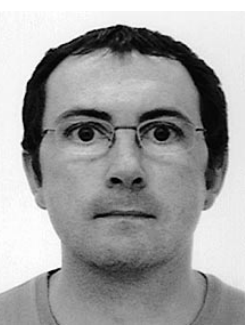

Benito Gimeno (M'01) was born in Valencia, Spain, on January 29,1964 . He received the Licenciado degree in physics and $\mathrm{Ph} . \mathrm{D}$. degree from the Universidad de Valencia, Valencia, Spain, in 1987 and 1992, respectively.

From 1987 to 1990, he was a Fellow with the Universidad de Valencia. Since 1990, he has been an Assistant Professor with the Departmento de Física Aplicada, Universidad de Valencia, where, in 1997, he became an Associate Professor. From 1994 to 1995, he was a Research Fellow with the European Space Research and Technology Centre (ESTEC), European Space Agency (ESA). His current research interests include computer-aided techniques for analysis of microwave passive components, waveguide structures including dielectric resonators, and photonic crystals. 